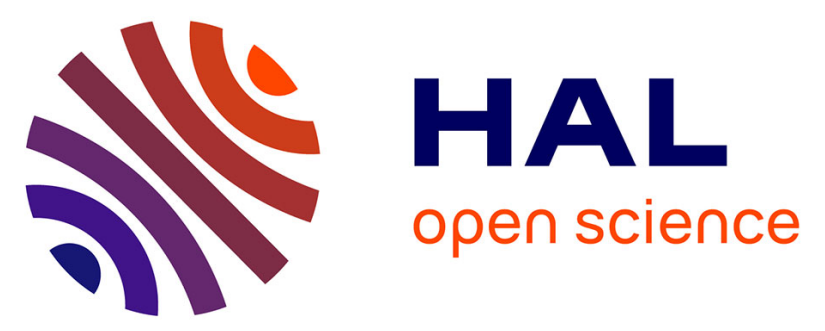

\title{
LOST: Localized blacklisting aware scheduling algorithm for IEEE 802.15.4-TSCH networks
}

Dimitrios Zorbas, Vassilis Kotsiou, Fabrice Theoleyre, Georgios Papadopoulos, Christos Douligeris

\section{- To cite this version:}

Dimitrios Zorbas, Vassilis Kotsiou, Fabrice Theoleyre, Georgios Papadopoulos, Christos Douligeris. LOST: Localized blacklisting aware scheduling algorithm for IEEE 802.15.4-TSCH networks. WD 2018 - 10th Wireless Days Conference, Apr 2018, Dubai, United Arab Emirates. pp.110-115, 10.1109/WD.2018.8361705 . hal-02336107

\section{HAL Id: hal-02336107 https://hal.science/hal-02336107}

Submitted on 29 Jan 2020

HAL is a multi-disciplinary open access archive for the deposit and dissemination of scientific research documents, whether they are published or not. The documents may come from teaching and research institutions in France or abroad, or from public or private research centers.
L'archive ouverte pluridisciplinaire HAL, est destinée au dépôt et à la diffusion de documents scientifiques de niveau recherche, publiés ou non, émanant des établissements d'enseignement et de recherche français ou étrangers, des laboratoires publics ou privés. 


\title{
LOST: Localized Blacklisting Aware Scheduling Algorithm for IEEE 802.15.4-TSCH Networks
}

\author{
Dimitrios Zorbas*, Vassilis Kotsiou ${ }^{\ddagger}$, Fabrice Théoleyre ${ }^{\ddagger}$, Georgios Z. Papadopoulos ${ }^{\dagger}$, and Christos Douligeris* \\ *NetLab, Department of Informatics, University of Piraeus, Greece, \{dzorbas,cdoulig\}@unipi.gr \\ ${ }^{\ddagger}$ CNRS, ICube, University of Strasbourg, France \{kotsiou, theoleyre\} @ unistra.fr \\ †IMT Atlantique, IRISA, UBL, France, georgios.papadopoulos@imt-atlantique.fr
}

\begin{abstract}
Industrial applications require more and more lowpower operations, low-delay, deterministic communications as well as end-to-end reliability close to $100 \%$. IEEE 802.15.4-TSCH (Time-Slotted Channel Hopping) relies on a channel hopping technique while scheduling properly the transmissions to provide a high end-to-end reliability. Because of external interference, some channels may perform very poorly locally, which impacts negatively the reliability for some radio links. We propose here the first distributed scheduling solution which reactively allocates the cells to each pair of nodes while also considering local blacklists. These local blacklists are constructed on a perradio link basis to reflect the actual performance encountered locally. Our simulations highlight the relevance of our distributed blacklisting aware scheduling algorithm to improve both the reliability and the delay efficiency compared with DeTAS, a state of the art distributed solution.
\end{abstract}

Index Terms-IoT; IEEE 802.15.4; TSCH; Distributed Scheduling; Interference; Blacklisting;

\section{INTRODUCTION}

Critical applications within the Industrial 4.0 era require efficient communication among the devices with end-to-end reliability close to $100 \%$ [1]. However, the constrained devices combined with the nature of wireless communications and the a priori unknown conditions over the deployment area, may present significant communication challenges and, thus, endanger the reliable data collection.

Slow channel hopping MAC such as IEEE 802.15.4TSCH [2] has been proposed to provide high-reliability at the link-layer. They rely on a strict organization of the different transmissions to eliminate the collisions. A schedule is constructed, such that two interfering transmitters are allocated to different timeslots. Besides, channel hopping allows the network to combat external interference [3]. While the medium access under $\mathrm{TSCH}$ relies on scheduling the transmissions, the actual algorithm to use is let unspecified in the standard. Therefore, several centralized and distributed scheduling algorithms have been proposed so far.

Because external interference may affect some specific channels, channel hopping may be insufficient: the transmissions using the bad channels impact negatively the reliability and the energy efficiency. With blacklisting (BL), the bad channels are identified and may be removed from the hopping sequence. By using only the most reliable channels, the reliability is globally improved. However, since a physical channel exhibits location-dependent characteristics [4], the blacklist is radio-link dependent, and should be updated locally. The idea of channel BL is not new since it has already used for other protocols, like WirelessHART. However, there is no localized mechanism to tackle local interference and it is assumed that it is a task globally performed by a systems administrator [5]. Thus, the localized approach presented in this paper may also be used in other communication standards.

To the best of our knowledge, we propose here the first complete localized scheduling algorithm combined with a reactive and localized BL mechanism.

The contributions of this paper are as follows:

1) we present a localized scheduling algorithm to assign the cells (matrix of channel offsets and timeslots) by exchanging information only with the neighbors;

2) we propose a distributed (i.e., per pair of nodes) BL scheme, that can modify its frequency hopping sequence to avoid the bad channels;

3) we use an adaptive over-provisioning scheme in the $\mathrm{TSCH}$ scheduler to increase the network reliability.

4) our performance evaluation highlights the relevance of this approach to increase the number of delivered packets while decreasing the end-to-end delay.

\section{RELATED WORK}

\section{A. IEEE 802.15.4-TSCH \& Radio Channel BL Techniques}

IEEE 802.15.4-2015 has proposed the TSCH mode, where the time is divided into timeslots of equal length. At each timeslot, a node may transmit or receive a frame, or it may turn to sleep mode for saving energy. A slotframe comprises a fixed set of timeslots, repeated cyclically. Each timeslot is labelled with an Absolute Sequence Number (ASN), which counts the number of timeslots since the network was established. Based on the ASN and the schedule, the nodes in the TSCH network decide when to transmit or receive a frame.

TSCH presents a deterministic scheduling approach in which each cell consists of a pair of timeslot and channel offset. The standard maintains a schedule, and assigns a set of cells to each radio link. At the beginning of a timeslot, the channel offset is translated into a physical channel using the ASN value:

$$
\text { frequency }=F((A S N+\text { channelOff set }) \% n F r e q)
$$

where channelOffset is the channel offset of the current cell, $n$ Freq is the number of available channels and $F()$ a 
bijective function mapping an int comprised between 1 and $n$ Freq into a physical channel [6].

BL consists in excluding the corresponding radio channels to be used for transmissions. To this aim, each pair of nodes must be able to identify accurately the bad channels which impact the reliability of its radio link. Detecting bad channels may use the average ETX (per channel) value [7], or any other link quality metric.

A blacklist may be applied globally in the whole network or locally using a per link method. Each of the two approaches has some advantages and disadvantages as it is discussed in [8]. In this paper we use a per link blacklisting approach.

\section{B. Scheduling algorithms for TSCH}

While 6TiSCH defines how the cells are negotiated, any scheduling algorithm may be actually implemented.

The centralized approaches rely on a Path Computation Element (PCE). TASA proposes to construct a schedule for a multihop TSCH network [9]. Dobslaw et al. [10] propose to reserve additional timeslots for retransmissions, in order to improve the reliability. MABO-TSCH assigns in a centralized manner a collection of cells for each radio link, while considering local blacklists [11]. Several channel offsets are assigned within the same timeslot, so that a radio link can pick one of the channel offset which does not give a blacklisted physical frequency.

Under distributed algorithms, the nodes exchange control packets only with their neighbors to detect and to eliminate the collisions. DeTAS proposes a decentralized version of TASA [12]. The children of the border routers collect the radio topology of their subtree to compute independently the schedule of their descendants (called micro-schedule). Wave [13] constructs a schedule such that a packet is delivered before the end of the slotframe, even if it has to be relayed by intermediate nodes. Hosni et al. [14] organize the transmissions into stratums to upper bound the end-to-end delay, even in presence of retransmissions. SF0 [15] represents the default behavior of $6 \mathrm{TiSCH}$. It reactively computes the number of cells to reserve based on the traffic it has to transmit. Overprovisionning and an hysteresis function help the network to be more stable and reliable. Furthermore, Orchestra was recently proposed [16] to construct a TSCH schedule in a distributed manner. Each node constructs pseudo-randomly its schedule without exchanging any control information with its neighbors.

Note that unlike some of the existing approaches our proposed approach guarantees that no collision between two or more transmissions occurs.

\section{BLACKLISTING AWARE SCHEDULING ALGORITHM}

In this Section, we present LOST (LOcalized Scheduling algorithm for TSCH networks), an efficient algorithm that establishes schedules and assigns channels for IEEE 802.15.4TSCH networks. LOST is a localized algorithm since it relies on information gathered by its 1-hop neighbors only.

LOST's features are summarized as follows:

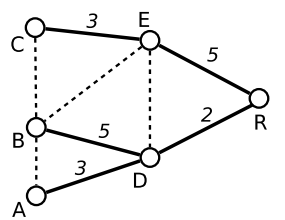

\begin{tabular}{lcccc}
\hline Timeslots & $1-5$ & $6-8$ & $9-18$ & $19-21$ \\
\hline Links & B-D & A-D & D-R & E-R \\
& E-R & C-E & & \\
\hline Channel offsets & 1 & 1 & 0 & 0 \\
& 0 & 0 & & \\
\hline
\end{tabular}

Fig. 1. Example topology and the corresponding LOST scheduling.

1) 1-hop only information is required. No centralized mechanism is involved in both slot allocation and offset assignment;

2) the algorithm multiplexes the different transmissions across different channels by allocating properly the channel offsets;

3) LOST prioritizes the nodes which have a larger number of packets in their queue. This action reduces the probability of buffer overflows, and decreases the delay;

4) No initial or global knowledge of the number of packets of the predecessor nodes is required. This makes the algorithm adaptive to topology changes;

5) A localized BL method is employed in the scheduler to avoid using the bad radio channels;

6) An over-provisioning scheme allocates additional timeslots for retransmissions within the same slotframe to bound the end-to-end delay.

We rely on IPv6 Routing Protocol for Low-Power and Lossy Networks (RPL) to construct the routes. LOST allocates the transmission opportunities along these routes to forward all traffic. Otherwise, a special mechanism needs to be introduced in LOST so that each node is aware of its parent. According to RPL, each node selects a preferred parent, from the list of possible parents, to which it will forward all its traffic. Each node is also aware of its rank in the RPL tree. Inversely, a node receives Destination Advertisement Object (DAO) control packets from its descendants, and forwards them to the border router through its own preferred parent. It exploits these DAO to maintain the list of its children. Each node in the network also acquires the information regarding the maximum degree of the DODAG (Destination Oriented Directed Acyclic Graph) during its construction. Finally, a node maintains a list of its physical neighbors, updated when it receives an Enhanced Beacon (EB).

The scheduling process of LOST consists of several rounds each of them comprising three phases. In each round, each node decides one of the three available states to follow depending on the state of its neighbors. LOST can run in an slotted or unslotted period of time. Collisions of control packets are avoided using priorities. Nodes with a higher priority transmit first. Due to the limited size of this paper, we omit the description of the collision avoidance mechanism.

We consider three types of states: the requester requests timeslots from its parent who will act as respondent. The respondent is in charge of assigning timeslots and replying to the requester. If a node has no request to send and at the same time it is not respondent, it remains in sleep mode. We 
will discuss about this last state later.

Next, we describe the phases of LOST and how a node individually decides if it will be requester or respondent. Note that the first two phases can be combined in a single phase (transmitting a single control packet), however, we separate the two phases for presentation purposes. To further facilitate the understanding of the algorithm, we introduce the example of Figure 1 consisting of 5 nodes (A-E) all of them transmitting packets to the root $(\mathrm{R})$. The solid lines correspond to the RPL links (node $\rightarrow$ parent) and the dashed lines to the physical radio links. The number above each link denotes the number of generated packets per node $\left(q_{i}\right)$.

\section{A. Dissemination of the Amount of Traffic}

The purpose of this phase is to let the neighboring nodes (parent and children) know about the amount of packets that each particular node intends to transmit. 1-hop information is exchanged among neighbors during this phase. The information that is exchanged includes the node id $i$, the amount of packets $q_{i}$ that exist in its (virtual) buffer and a priority number prior $_{i}$. LOST gives priority to the nodes that transmit a higher number of packets and are close to the root in order to avoid buffer overflows. Thus, prior $_{i}$ is computed as a function of $q_{i}$ as follows: prior $_{i}=\frac{q_{i}}{r_{i}}+\epsilon$, where $r_{i}$ is the rank of node $i$ and $\epsilon$ is a real very small random number. In our example, nodes $\mathrm{B}$ and $\mathrm{E}$ have the highest priorities.

\section{B. Slots Requests}

Every node that receives the neighboring piece of information described in the previous phase can now decide if it will be a requester or respondent in this round. A node can be a requester if it has a higher priority than its parent. A requester sends its request to its parent using its id, priority, the number of requested slots, and the last already assigned timeslot (if any). In our example, nodes $\mathrm{A}, \mathrm{B}$, and $\mathrm{E}$ will be requesters and $\mathrm{D}, \mathrm{R}$ will be respondents. Finally, $\mathrm{C}$ will wait the next round to request slots.

\section{Slots Allocation}

Finally, a respondent will rank the requests according to their priority, starting from the highest. It will then check its first available slot and will assign a number of slots equal to the requested slots for each of the (priority-ranked) requested children. A reply is sent to its children with the allocated slots. In the example of Figure 1, D will allocate slots 1-5 for B and slots 6-8 for $\mathrm{A}$. On the other hand, $\mathrm{R}$ will allocate slots 1-5 for $\mathrm{E}$.

When a node receives a reply from its respondent, it sets its $q$ value to 0 . On the other hand, the respondent increases its $q$ value accordingly: a given flow will reserve hop by hop a sufficient amount of cells. This 3-step procedure is repeated until all the nodes have allocated a number of slots at least equal to the number of packets of their predecessor nodes plus their initial packets. Note that each node is aware of this information as the algorithm evolves from the leaf nodes to the root. A node knows that its allocation role is completed in the algorithm if it does not receive any other requests within the next round from the time it set its $q$ value to 0 .

\section{Channel offset assignment}

We have now to assign the channel offsets so that two interfering nodes which selected the same timeslot use a different channel offset. We consider that a collision may arise because (i) at least one node from one link is physical neighbor with at least one node of the other link, or (ii) the sender of one link is 2-hop RPL neighbor with the sender of the other link.

LOST follows a distributed approach to assign the channel offsets and avoid the collisions. Initially all the nodes have assigned a channel offset equal to 0 . Each node follows a twostep procedure as soon as it has already allocated the timeslots for its packets. A node has the right to decide which channel offset to use, when it has already received the channel offsets of all its predecessor RPL nodes. This means that the channel assignment is a recursive procedure that starts from the leaf nodes and moves on to the root.

During the first step, we keep on exploiting the local priorities to solve the conflicts with the physical neighbors. A node extracts the list of timeslots already reserved by its neighbors with an higher priority. If the timeslots reserved by the node, and these higher priority neighbors are the same, it has to select a different channel offset. Thus, it selects the next available offset (e.g., B will choose the channel offset 1 in figure 1). By exploiting the set of priorities, we are sure to order the decisions, and that only one node will change its schedule when a conflict is detected.

The second step ensures that no 2-hop neighbors transmit at the same time with the same offset. To achieve this, a node checks the reservations received from its children (which contain also the reservations of the grand-children). Similarly, a node checks if the same timeslots have been already reserved by one of these 2-hop neighbors. If an overlap is detected, the node selects a different channel offset. Once a node has reserved its cells (timeslots and channel offset) and has already completed the two steps, it can safely turn to the sleep mode state. We must note that if no other offsets are available due to a high node density in a neighborhood, a node can postpone its transmission in the current timeslot and request a new timeslot from its parent. However, this mechanism is not implemented in the current version of the algorithm.

\section{E. Adaptive timeslot provisioning}

LOST allocates additional timeslots per link in order to increase the communication reliability and to bound the endto-end delay in the case of failures. These extra timeslots are used as backup slots giving the opportunity to the sender to retransmit a packet that was not acknowledged previously. The strong point of LOST's over-provisioning mechanism is that it is adaptive to network traffic demands and communications failures.

In LOST, the number of extra timeslots depends on the Packet Error Rate (PER) between the sender and the receiver. 
PER can be computed by evaluating the link quality at the beginning of the process. Note, that if PER is not available, other link quality estimators can be used like the RSSI and the SNR. Eq. (2) describes the extra amount of slots $q_{i}^{P R}$ that will be requested by the requester $i$ during the second phase of the algorithm.

$$
q_{i}^{P R}=i n t\left(\alpha_{i}\left(\frac{P E R_{i j}}{m a x_{-} P E R}\right)^{2} q_{i}\right),
$$

where $\alpha_{i}$ is a constant that describes the necessity of the provisioning $\left(0 \leq \alpha_{i} \leq 1\right), P E R_{i j}$ is the packet error rate of transmitter $i$ and receiver $j$, and max_PER is the maximum error packet rate. Note that $\alpha_{i}$ is a node-dependent constant and it characterizes the link quality between $i$ and its parent. $\alpha_{i}$ can be modified in future executions of the algorithm (reschedules) according to the number of extra slots that were actually used during the previous slotframes. In particular we update $\alpha_{i}$ as follows:

$$
\alpha_{i}=\frac{\alpha_{i} F S+\frac{\text { reserved_slots_unused_slots }}{\text { reserved_slots }}}{F S+1},
$$

where reserved_slots is the number of slots reserved for transmitting $q$ packets ( $q \leq$ reserved_slots), unused_slots is the number of assigned slots that have finally not used, and $F S$ is the number of the frameslot.

\section{F. Overhead}

LOST exhibits a low overhead mainly because 1-hop only information is exchanged. The number of exchange messages per round depends on the number of participating nodes. In fact, $N-1$ messages are needed for the first two phases of the algorithm, where $N$ is the number of nodes including the sink. Moreover, each parent replies to its children requests sending a message with the schedule to each child. Finally, for the offset assignment two messages are required per node (excluding the sink); one for the 1-hop physical neighbors and one to forward the reservation to the parent. Thus, the total number of exchange messages per round is $4 N-3$.

We must note here that the number of exchange messages can be highly reduced if we assume that each node waits for the reception of the schedules of its children before allocating its own timeslots. However, this could dramatically increase the number of data packets in the buffer, causing buffer overflows, delays, and longer schedules.

\section{G. Blacklisting Aware Channel Hopping}

At the beginning of each timeslot, an active node (either transmitter or receiver) has to select the actual physical frequency to use. The standard specifies it is derived from the channel offset and the ASN using the Eq. 1 (cf. section II-A). A pair of nodes has to agree on the blacklist to use: inconsistent blacklists may make the receiver deaf, impacting negatively the reliability. On the other hand, even if two parallel links have different channel offsets, eq. (1) may still generate the same physical frequency for both links from time to time in absence of a BL mechanism.

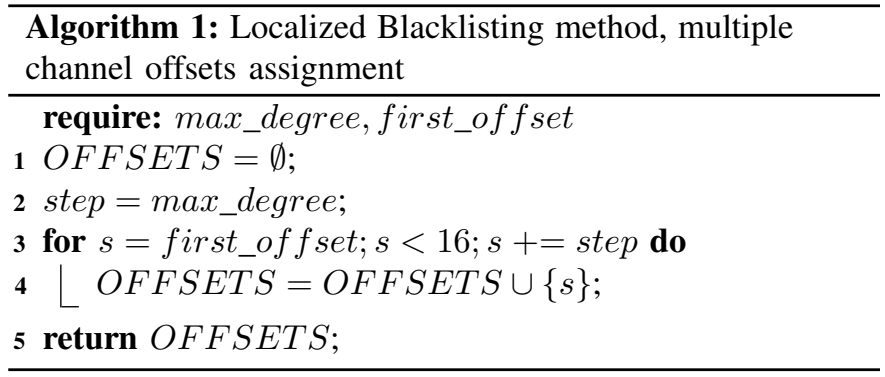

To tackle this problem, two solutions are possible: either globally blacklisting bad channels or the list of blacklisted channels is exchanged locally. The disadvantage of the first case is that all the nodes must have a consistent view of the blacklists used by the other ones, thus, the information needs time to be propagated to the whole network. Moreover, extra messages need to be injected in the network increasing the energy cost.

In this paper, we use a localized BL method to keep bad channels locally without distributing them to the entire network. This method enhances the channel offset assignment phase described in Section III-D by allowing a node to assign multiple offsets per link. This process is described in Algorithm 1. In particular, every node keeps track of a set of available channel offsets (i.e., OFFSETS) generated by a simple function based on the maximum vertex degree in network. The maximum vertex degree is used so that an adequate number of channel offsets is produced even for high density areas (areas with possibly many parallel links) [17]. If first_offset (first_offset $\in[0,15]$ ) is the channel offset assigned by the channel offset process of Section III-D, a few other offsets are generated with distance step with each other. Since first_offset is different for each parallel link and step (as well as nFreq) is the same for all the nodes, Eq. 1 always generates different channels for all parallel links. If the generated channel with one offset is blacklisted, a new channel is generated using the next available offsets until a whitelisted channel is finally produced. If none whitelisted channel is generated using all the possible offsets, the node postpones its transmission.

Note that each pair of nodes must retain the same blacklist to communicate properly. To do so, neighboring nodes can encapsulate blacklists in a data or in an acknowledgement packet without increasing considerably the payload and thus the energy consumption [11].

Thus, B and A of Figure 1 will assign offsets 1, 5, 9, and 13 , while the rest of the nodes will assign $0,4,8$, and 12 .

\section{Performance Evaluation}

In this Section, we evaluate the LOST algorithm and we compare its performance against DeTAS [12]. We use 4 flavors of LOST; one with both BL and provisioning (designated as LOST), one with BL and without provisioning (LOST-NPR), one with provisioning and without BL (LOST-NBL), and one without BL or provisioning (LOST-NBL-NPR). The purpose 
of distinguish these four flavors is to assess the affect of BL and over-provisioning schemes to the algorithm performance.

The results are obtained using a set of Monte Carlo simulations with a simulator written in Perl programming language. The topologies were generated using the DeTAS' terrain generator with a $200 \times 200 \mathrm{~m}^{2}$ terrain size, random node positions, and a communication range of $50 \mathrm{~m}$ [12]. This implies an average number of neighbors per node between 2 and 9. Each node generates a random number of packets per slotframe in the range [1,5]. Due to the high number of generated packets, the length of the schedule may be higher than the 101 timeslots described by the IEEE 802.15.4 TSCH standard (even without provisioning). Therefore, we have increased the number of available timeslots per slotframe to 301 . Moreover, we use a probability $P$ that a packet is dropped due to external interference for each of the 16 available channels. The following values were used: $P_{11}=0.3, P_{12}=0.4, P_{13}=0.4$, $P_{14}=0.3, P_{15}=0.01, P_{16}=0.3, P_{17}=0.4, P_{18}=0.4, P_{19}=0.01$, $P_{20}=0.01, P_{21}=0.2, P_{22}=0.4, P_{23}=0.4, P_{24}=0.01, P_{25}=0.01$, and $P_{26}=0.01$. Finally, we set $\alpha=0.5$ for all the nodes. A channel is blacklisted if the Packet Delivery Ratio (PDR) falls bellow 0.9. We vary the number of nodes and we measure the PDR and the total packets delayed within 50 slotframes.

1) PDR: In Figure 2, the network-wide PDR performance under various densities is illustrated. As it can be observed, the proposed LOST scheme achieves PDR above 99\%, more than any other LOST configuration or DeTAS scheme. The results demonstrate the significant adding value of both $\mathrm{BL}$ and over-provisioning mechanisms, i.e., LOST-NBL-NPR presents a PDR below $80 \%$. It is worth mentioning that LOST outperforms DeTAS even without these mechanisms. This is mainly because of the low channel diversity of DeTAS which results to higher number of collisions.

2) Delay: Next, we evaluate the delay performance of LOST scheme. For successfully delivered packets, LOST's end-to-end delay is at the same level as that of DeTAS. However, on average it is considerably affected by failures on the path to the sink. We assume that a packet is delayed if it was not delivered within an interval of one slotframe. Our simulation results show that the network density and, consequently, the additional traffic in the network do not impact negatively LOST in terms of delay. As it can be observed from Figure 3, LOST presents a very low and stable performance. On the other hand, the results show that without employing BL and over-provisioning, LOST-NBL-NPR linearly degrades its performance with increase of density. Finally, DeTAS once again presents very poor results, it is straightforward that it can not handle high level of traffic in the presence of interference.

3) Impact of Over-provisioning: We study here the impact of over-provisioning method on the size of LOST's schedule. Note that in this case $\alpha$ is initially set to 0.5 and it is updated according to Eq. 3. Figure 4 illustrates LOST's schedule size after the first execution, at the end (i.e., re-scheduling after 50 slotframes), and without over-provisioning. As it can be observed, after 50 slotframes, the schedule converges and adapts to the requested traffic conditions. Moreover, the more



Fig. 2. Packet Delivery Ratio for different node populations.



Fig. 3. Number of delayed packets for different node populations.

dense is the network, the more efficient is the converged schedule. Indeed, in case of 50 nodes in the network, the size of the schedule after re-scheduling and without overprovisioning is similar. As a result, this evaluation shows the adaptability of our method and the minimum impact of overprovisioning on the schedule, while improving essentially the network performance in terms of PDR and delay.

4) Impact of $\alpha$ : We focus here on the impact of $\alpha$ on the network reliability. To this aim, we evaluate the PDR performance of LOST scheme with and without over-provisioning over $\alpha$. We present two different use-cases: $i$ ) each node transmits on average three packets per slotframe (i.e., slotframe size of 301 timeslots), ii) each node transmits on average one packet per slotframe (i.e., slotframe size of 101 timeslots). As it was expected, LOST without over-provisioning, presents stable PDR performance, since it has no impact from $\alpha$. On the other hand, low $\alpha$ values do not allow many packet retransmissions leading to a slightly lower PDR compared to middle-range values. On the contrary, large $\alpha$ values lead to very long schedules increasing the number of nodes that are unable to send their packets within the desired slotframe.

5) Overhead: Finally, Figure 6 presents the overall LOST's overhead needed to build a schedule for different node populations. We can observe that the number of messages increases linearly with the number of nodes. About 6 messages are needed per node to build the schedule (allocate slots and channels offsets).

\section{COnclusions \& Future Work}

We presented here a distributed scheduling solution, which allocates the timeslots and channel offsets. We assign a set of priorities so that a cell is reserved by the highest priority node, 


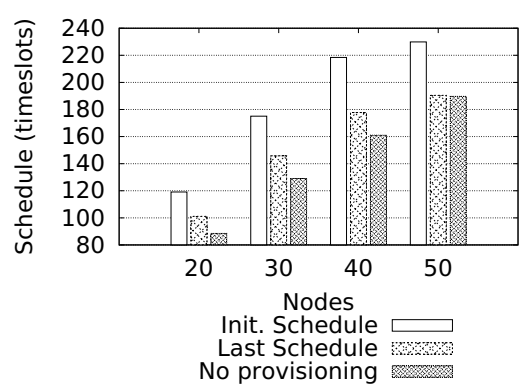

Fig. 4. Schedule lengths before and after rescheduling.

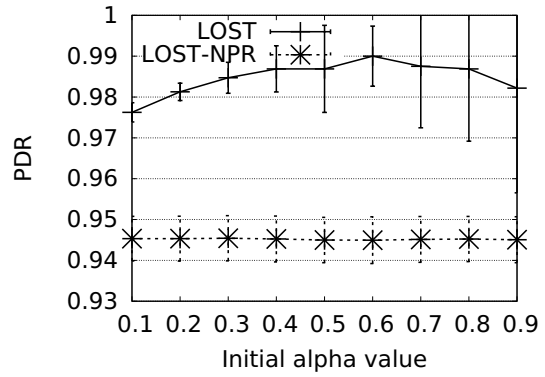

(a) 3-packets per node on average (slotframe size $=301$ timeslots)

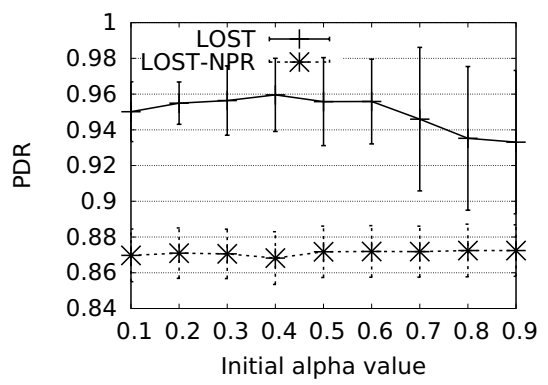

(b) 1-packet per node (slotframe size $=101$ timeslots)

Fig. 5. PDR for different $\alpha$ values and slotframes sizes.

which locks its usage in its neighborhood. Besides, we also combine a BL technique to not use the bad channels, which provide a poor reliability. Our simulations highlight the superiority of this approach compared with DETAS, a reference solution for distributed scheduling in TSCH. By avoiding both collisions and the usage of the locally bad channels, LOST is able to multiplex efficiently the transmissions and to improve the network reliability. In the future, we plan to evaluate the performance of LOST in a testbed, where the conditions are dynamic. We conjecture that our over-provisioning and BL mechanisms will help to guarantee high-reliability.

\section{ACKNOWLEDGEMENTS}

This work was carried out within the action "Strengthening Post Doctoral Research" of the "Human Resources Development Program, Education and Lifelong Learning", 2014-2020, which is being implemented from IKY and is co-financed by the European Social Fund - ESF and the Greek government.

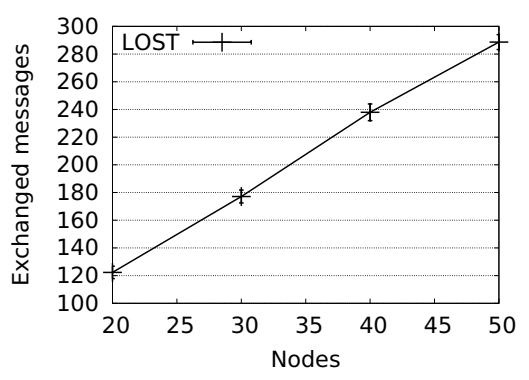

Fig. 6. LOST's overhead for different node populations.

It has also received funding from the European Union's Horizon 2020 Research and Innovation Programme under Grant Agreement No 740477 - SAURON.

\section{REFERENCES}

[1] L. D. Xu, W. He, and S. Li, "Internet of things in industries: A survey," IEEE Transactions on Industrial Informatics, vol. 10, no. 4, pp. 22332243, Nov 2014.

[2] "IEEE Standard for Low-Rate Wireless Personal Area Networks (LRWPANs)," IEEE Std 802.15.4-2015 (Revision of IEEE Std 802.15.42011), April 2016.

[3] T. Watteyne, A. Mehta, and K. Pister, "Reliability through frequency diversity: Why channel hopping makes sense," in PE-WASUN. ACM, 2009.

[4] V. Kotsiou, G. Z. Papadopoulos, P. Chatzimisios, and F. Theoleyre, "Is Local Blacklisting Relevant in Slow Channel Hopping Low-Power Wireless Networks?” in ICC. IEEE, 2017.

[5] S. Petersen and S. Carlsen, "Wirelesshart versus isa100.11a: The format war hits the factory floor," IEEE Industrial Electronics Magazine, vol. 5, no. 4, pp. 23-34, Dec 2011.

[6] T. Watteyne, M. Palattella, and L. Grieco, "Using IEEE 802.15.4e Time-Slotted Channel Hopping (TSCH) in the Internet of Things (IoT): Problem Statement," RFC 7554, 2015.

[7] M. Sha, G. Hackmann, and C. Lu, "Arch: Practical channel hopping for reliable home-area sensor networks," in RTAS. IEEE, 2011.

[8] D. Zorbas, G. Z. Papadopoulos, and C. Douligeris, "Local or global radio channel blacklisting for ieee 802.15.4-tsch networks?" in IEEE International Conference on Communications (ICC). IEEE, Apr 2018.

[9] M.R. Palattella, et al., "On optimal scheduling in duty-cycled industrial iot applications using IEEE802.15.4e TSCH,' Sensors Journal, IEEE, vol. 13 , no. 10 , pp. 3655-3666, Oct 2013.

[10] F. Dobslaw, T. Zhang, and M. Gidlund, "End-to-end reliability-aware scheduling for wireless sensor networks," IEEE Transactions on Industrial Informatics, vol. 12, no. 2, pp. 758-767, April 2016.

[11] P. H. Gomes, T. Watteyne, and B. Krishnamachari, "MABO-TSCH: Multihop and blacklist-based optimized time synchronized channel hopping," Transactions on Emerging Telecommunications Technologies, August 2017.

[12] N. Accettura, M. Palattella, G. Boggia, L. Grieco, and M. Dohler, "Decentralized traffic aware scheduling for multi-hop low power lossy networks in the internet of things," in WoWMoM. IEEE, 2013, pp. 1-6.

[13] R. Soua, P. Minet, and E. Livolant, "Wave: a distributed scheduling algorithm for convergecast in IEEE 802.15.4e TSCH networks," Transactions on Emerging Telecommunications Technologies, vol. 27, no. 4, pp. 557-575, Oct. 2016.

[14] I. Hosni and F. Théoleyre, "Self-healing distributed scheduling for endto-end delay optimization in multihop wireless networks with 6tisch," Computer Communications, vol. 110, pp. 103 - 119, 2017.

[15] D. Dujovne, L. A. Grieco, M. R. Palattella, and N. Accettura, "6tisch 6top scheduling function zero (sf0)," IETF, draft 4, July 2017, https: //tools.ietf.org/html/draft-ietf-6tisch-6top-sf0-04.

[16] S. Duquennoy, B. Al Nahas, O. Landsiedel, and T. Watteyne, "Orchestra: Robust mesh networks through autonomously scheduled tsch," in Sensys. ACM, 2015, pp. 337-350.

[17] B. Bollobás, "Chromatic number, girth and maximal degree," vol. 24, pp. 311-314, 1978. 